\title{
Investigating dense nuclear matter with rare hadronic probes
}

\author{
Manuel Lorenz for the HADES collaboration* \\ Goethe University Frankfurt, Max-von-Laue-Str.1 60438 Frankfurt am Main, Germany \\ E-mail: Lorenz@Physik.uni-frankfurt.de
}

We report on HADES measurements of strange hadrons in collisions of $\operatorname{Ar}(1.76 \mathrm{AGeV})+\mathrm{KCl}$. Besides $K^{+}$and $K_{S}^{0}$ mesons, for the first time at this energy, a combined identification of subthreshold $K^{-}$and $\phi$ mesons was carried out. Subthreshold strange particle production is believed to be a strongly associated process due to strangeness exchange reactions therefore it is important to measure as many particles as possible containing strangeness to achieve a complete picture. The high yield of the $\phi$ meson, $(2.6 \pm 0.7 \pm 0.1) \times 10^{-4}$, points to its importance as a source for the $K^{-}$mesons, while the observation of the doubly strange $\Xi^{-}$hyperon more than $600 \mathrm{MeV}$ below its free NN production threshold rises questions on the understanding of subthreshold production of strangeness. We investigate the consequences of the high $\phi$ yield for the slope of the $K^{-}$, taken as a hint for different freeze out criteria of kaons and antikaons, and find that the different slopes can be explained by a simple superposition of directly produced $K^{-}$and such ones stemming from $\phi$ decays.

XLVIII International Winter Meeting on Nuclear Physics, BORMIO2010

January 25-29, 2010

Bormio, Italy

\footnotetext{
*Speaker.
} 


\section{Strangeness in dense nuclear matter}

Heavy-ion collisions at kinetic beam energies of 1-2 AGeV create a rather dense (compression factors up to three) and long-lived system (lifetimes in the order of $10 \mathrm{fm} / \mathrm{c}$ ) [1], providing a favorable environment to study the hadron properties under such extreme conditions. According to theoretical calculations antikaons feel an attractive potential in the nuclear medium, whereas the in-medium kaon potential is expected to be slightly repulsive [2]. These effects are expected to affect the production and propagation of kaons in dense nuclear matter, yielding a large mean free path and reduced production for kaons and a increased production but small mean free path for antikaons. Consequently, the systematic study of $K^{ \pm}$sub-threshold production yields, phase space distributions and flow observables attracted much experimental attention in the past two decades. A major experimental finding of the KaoS collaboration is that the $K^{-} / K^{+}$ratio is almost constant as a function of the collision centrality. The inverse slope parameters of the energy distributions of $K^{+}$are systematical about 15 to $25 \mathrm{MeV}$ larger than those of the $K^{-}$. The following conclusions are drawn in [3]. The $K^{-}$and the $K^{+}$yields are coupled by a strangeness exchange reaction e.g.

$$
\pi Y \longrightarrow K^{-} N
$$

(with Y: $\Lambda$ or $\Sigma$ ) and both particle species exhibit different freeze-out conditions. This is in accordance with transport model calculations which predict different emission times for $\mathrm{K}^{+}$and $\mathrm{K}^{-}$ mesons due to this process [4]. These findings need to be confronted with statistical model calculations which are also able to describe the constant $\mathrm{K}^{-} / \mathrm{K}^{+}$ratio assuming unique freeze-out conditions [5].

\section{HADES results on strangeness production in HIC}

The observed associated production of strange mesons underlines the need for a simultaneous measurement of all major particles containing strangeness in order to achieve a complete picture of their production and propagation. The HADES spectrometer at the heavy-ion synchrotron SIS at the Helmholtzzentrum für Schwerionenforschung (GSI) in Darmstadt has besides lepton identification power, the ability for precise measurements of nearly all relevant hadrons containing strangeness [6]. Besides $K^{+}$and $K_{S}^{0}$ mesons, a combined identification of $K^{-}$and $\phi$ mesons was carried out in the collision system $\mathrm{Ar}+\mathrm{KCl}$ at $1.76 \mathrm{AGeV}$ for the first time at this energy. Using the symmetry of the collision system the rapidity coverage of charged kaons is around $70 \%$. As the ratio of $K^{-} / K^{+}$is nearly independent of centrality it can be directly compared to the ones measured by KaoS at different energies in various systems, see left panel of figure 1 . While the charged kaon data fit well into the obtained systematics, the unexpectedly high yield of the $\phi$ meson of $(2.6 \pm 0.7 \pm 0.1) \times 10^{-4}$, translates into $0.18 \pm 7$ of all $K^{-}$mesons originate from $\phi$ decays and underlines its importance as a source for the $K^{-}$mesons, as already indicated by data of the FOPI collaboration [7]. A fact which is so far taken into account only by one transport calculation [8].

Another discovery of HADES is the identification of the doubly strange $\Xi^{-}$hyperon more than $600 \mathrm{MeV}$ below its free NN production threshold. So far, both transport and statistical models fail by more than order of magnitude to describe these data, which seems to question the understanding of subthreshold strangeness production. Both findings and details of the data analysis are discussed 

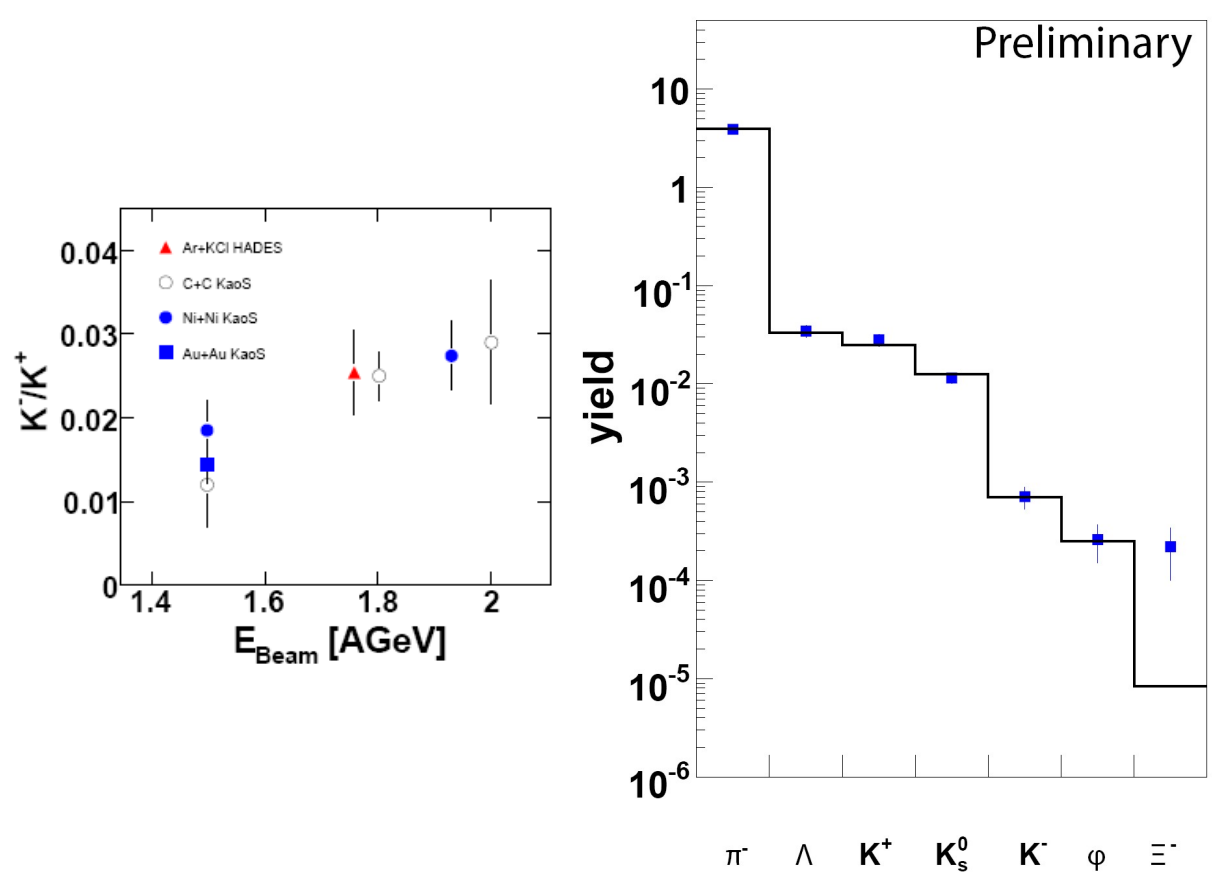

Figure 1: Left panel: $K^{-} / K^{+}$ratios as a function of the kinetic beam energy for various systems. The present HADES data point [9] is depicted by the full triangle. KaoS data (open and filled circles, full square) are from [3]. Right panel: Comparison of measured particle yields from [9, 10, 11, 14] to a statistical model fit.

in [9] and [10]. The measurement of $K_{S}^{0}$ mesons down to very low transverse momentum provides an excellent option to study the in-medium potential of kaons in comparison to transport models, as the effect should be most visible for those particles emitted with low momentum in the center of mass system of the reaction. As the ratio of antikaons to kaons is small, the measured $K_{S}^{0}$ experienced except for the Coulomb interaction, nearly the same slightly repulsive in-medium potential as the $K^{+}$mesons. More details can be found in [11]. The measured particle multiplicities of $\pi^{-}, \Lambda, K^{+}, K_{S}^{0}, K^{-}, \phi, \Xi^{-}$are used as input for a statistical model fit using the Thermus code [12]. In this model a strangeness correlation radius $R_{c}$ [13] in which strangeness is exactly conserved besides temperature and chemical potential parameters are adjusted to the data. The statistical model is able to reproduce the measured particle yields within errors, except for the $\Xi^{-}$hyperon, as already mentioned, see right panel of figure 1 . The following values for the chemical freeze-out conditions are extracted from the fit: $T \approx 73 \mathrm{MeV}, \mu_{b} \approx 770 \mathrm{MeV}, R \approx 4.9 \mathrm{fm}$ and $R_{c} \approx 2.4 \mathrm{fm}$. More details can be found in [14].

\section{On the different freeze out criteria of charged kaons}

Let us investigate the impact of the obtained large $\phi / K^{-}$ratio on the measured inverse slope parameter of antikaons, which was interpreted [3] as a sign for the later freeze out of this kaon species. We start our investigation with a simple assumption. Let us assume that there are only 
two sources of $K^{-}$, a thermal one, the other one feeded by the decay of $\phi$ mesons. In experiment one measures only the total yield. Using the event generator PLUTO [15] we generate a cocktail of $K^{-}$arising from the decay of purely thermal $\phi$ mesons according to the measured inverse slope of $T_{\phi}=84 \pm 8 \mathrm{MeV}$ and from a purely thermal source of $T_{K^{-}}=T_{K^{+}}=89 \pm 1 \pm 2 \mathrm{MeV}$ corresponding to the measured inverse slope of the positive kaons. In order to get realistic particle ratios we normalize the rapidity density function of the thermal $K^{-}$and the ones resulting from a $\phi$ decay according to the measured $\phi / K^{-}$ratio. The resulting distributions are shown in the left panel of figure 2 . In the next step we scale the transverse mass distribution around beam rapidity according
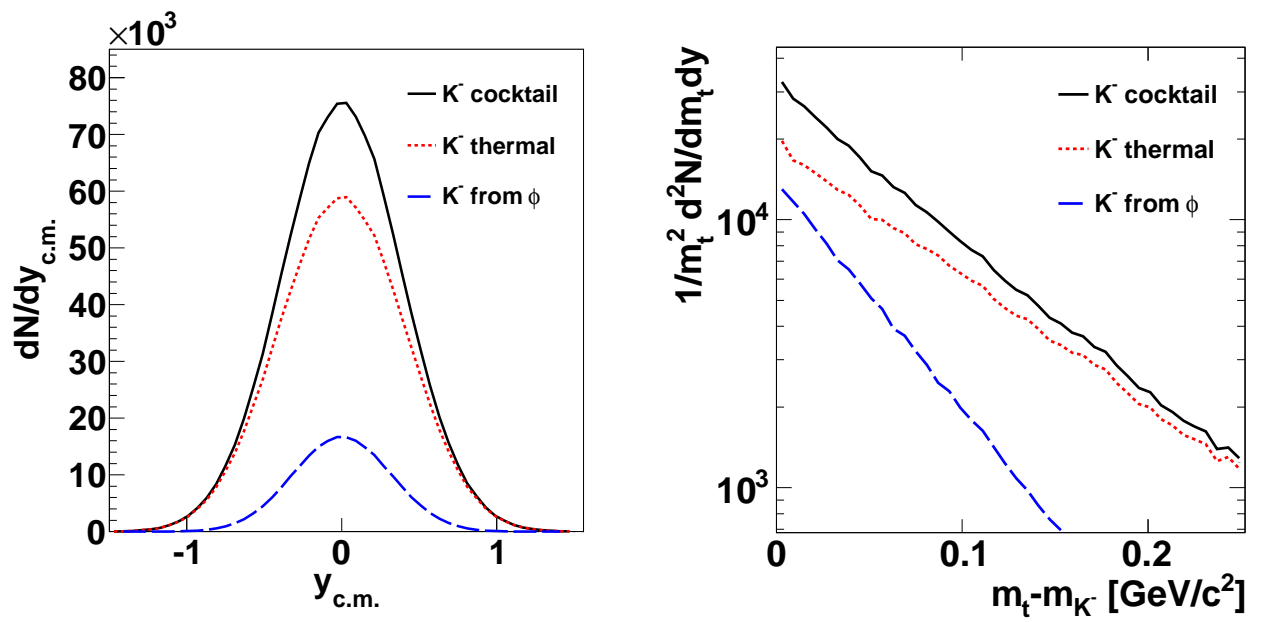

Figure 2: Left panel: According to the measured yields in [9] normalized rapidity density distributions of the thermal $K^{-}$and the ones resulting of a $\phi$. The black solid line shows the sum of the two distributions. Right panel: Simulated transverse mass spectra for $K^{-}$coming from a thermal source with a temperature of $89 \mathrm{MeV}$ and those which stem from a $\phi$ decay. The resulting cocktail spectrum is shown as a black solid line.

to the rapidity density functions. A Boltzmann fit according to

$$
\frac{1}{m_{t}^{2}} \frac{d^{2} N}{d m_{t} d y}=C(y) \exp \left(-\frac{\left(m_{t}-m_{0}\right) c^{2}}{T_{B}(y)}\right) .
$$

is applied in the HADES $m_{t}-m_{K}$ acceptance range $0-200 \mathrm{MeV} / \mathrm{c}^{2}$ for $\mathrm{K}^{-}$in the same way as it was done for the HADES data. The thermal $K^{-}$spectrum, the spectrum of the $K^{-}$which stem from the $\phi$ decay and the resulting cocktail spectrum are shown in the right panel of figure 2 . The inverse slope parameter of the $K^{-}$cocktail was found to be $T_{K^{-}}=74 \mathrm{MeV}$ and agrees within errors with the experimental obtained value of $T_{K^{-}}=69 \pm 2 \pm 4 \mathrm{MeV}$, leaving little room for possible different freeze-out conditions. With the assumption of two different production channels for the $K^{-}$it seems to be possible to describe the deviation of the inverse slope parameter of the charged kaons quite satisfactorily. This is in agreement with the statistical model assumption of unique freeze out criteria. For $K^{+}$the effect is negligible due to the much higher production yield, due to additional production channels as $N N \rightarrow N \Lambda K^{+}$. This effect is similar as the one observed for pions where two different slopes can be extracted from the transverse mass distributions. Here it is generally accepted that the origin of the smaller slope is the decay of a $\Delta$ resonance into $N \pi$. 


\section{Conclusion and outlook}

We show that the different inverse slope parameters of the kaon and antikaon do not necessarily point to different freeze-out criteria for the two kaon species, but can be explained by a simple superposition of decay kinematics when taking the new $\phi$ data into account. New measurements with higher statistic, increased particle identification power and acceptance should be able to distinguish between a pure Boltzmann like spectra or a superposition of two slopes similar to the case of the pions. High statistic measurement in an increased acceptance of kaons and $\phi$ mesons will be provided by HADES after the ongoing upgrade with high resolution RPC detectors and an up-toten times faster DAQ system which will be completed this year. An already installed forward wall hodoscope will allow for event plane reconstruction and flow observable analysis on top. With this setup it is planned to take data using medium and heavy sized ion collision systems ( $\mathrm{AgAg}, \mathrm{AuAu}$ ) from late 2010 on.

The collaboration gratefully acknowledges the support by BMBF grants 06DR135, 06MT238 T5, and 06MT9156 TP5 (Germany), by the DFG EClust 153, by GSI TMKRUE, by grants MSMT LC07050 and GA ASCR IAA100480803 (Czech Rep.), by grants RII3-CT-2005-515876, FPA200609154, and CPAN:CSD2007-00042(Spain).

\section{References}

[1] C. Hartnack, H. Oeschler and J. Aichelin, Phys. Rev. Lett. 90, 102302 (2003) [arXiv:nucl-th/0109016].

[2] J. Schaffner, A. Gal, I. N. Mishustin, H. Stoecker and W. Greiner, Phys. Lett. B 334, 268 (1994).

[3] A. Förster et al. (KaoS), Phys. Rev. C 75 (2007) 024906.

[4] C. Hartnack, H. Oeschler, J. Aichelin, Phys. Rev. Lett. 90 (2003) 102302.

[5] J. Cleymans, H. Oeschler and K. Redlich, Phys. Rev. C 59 (1999) 1663 [arXiv:nucl-th/9809027].

[6] G. Agakishiev et al. [HADES Collaboration], Eur. Phys. J. A 41 (2009) 243 [arXiv:0902.3478 [nucl-ex]].

[7] A. Mangiarotti et al. (FOPI), Nucl. Phys. A 714 (2003) 89.

[8] H. Schade, G. Wolf and B. Kampfer, Phys. Rev. C 81, 034902 (2010) [arXiv:0911.3762 [nucl-th]].

[9] G. Agakishiev et al. [HADES Collaboration], Phys. Rev. C 80 (2009) 025209 [arXiv:0902.3487 [nucl-ex]].

[10] G. Agakishiev et al. [HADES collaboration], Phys. Rev. Lett. 103 (2009) 132301 [arXiv:0907.3582 [nucl-ex]].

[11] G. Agakishiev et al., arXiv:1004.3881 [nucl-ex].

[12] S. Wheaton and J. Cleymans, Comput. Phys. Commun. 180 (2009) 84 [arXiv:hep-ph/0407174].

[13] I. Kraus, J. Cleymans, H. Oeschler, K. Redlich and S. Wheaton, Phys. Rev. C 76 (2007) 064903 [arXiv:0707.3879 [hep-ph]].

[14] G. Agakishiev et al. (HADES), "Hyperon production in $\mathrm{Ar}+\mathrm{KCl}$ reactions at $1.76 \mathrm{GeV} / \mathrm{u}$," to be published.

[15] I. Fröhlich et al., PoS ACAT2007 (2007) 076 [arXiv:0708.2382 [nucl-ex]]. 